\title{
Statistical Analysis and the OPEA Model of the White-Light Flares Occurring on Krüger 60B (DO Cep)
}

\author{
Hasan Ali Dal \\ Department of Astronomy and Space Sciences, University of Ege, Bornova, 35100 İzmir, Turkey. \\ Email: ali.dal@ege.edu.tr
}

\begin{abstract}
In this study, new observations and some results of statistical analyses are presented. The largest flare data set of DO Cep in the literature has been obtained with 89 flares detected in 67.61 hours of $U$-band flare patrol. First of all, the observations demonstrated that the star is one of the most active flare stars in respect to the computed flare frequency. Secondly, using the independent samples $t$-test, the detected flares were classified into two subtypes, and then they were modelled. Analysing the models demonstrated that the fast and slow flares occurring on the star can be separated with a critical value of the ratio of their decay time to rise time. The critical value was computed as 3.40. According to this value, the fast flare rate is $20.22 \%$, while the slow flare rate is $79.78 \%$. Besides, there is a 39.282 times difference between the energies of these two types of flares. However, the flare equivalent durations versus the flare rise times increase in similar ways for both groups. In addition, all the flares were modelled with the one-phase exponential association function. Analysing this model, the plateau value was found to be 2.810. Moreover, the half-life value was computed as $433.1 \mathrm{~s}$ from the model. The maximum flare rise time was found to be $1164 \mathrm{~s}$, while the maximum flare total duration was found to be $3472 \mathrm{~s}$. The results of the flare timescales indicate that the geometry of the flaring loop on the surface of the star might be similar to those seen on analogues of DO Cep. Consequently, considering both the half-life value and flare timescales, the flares detected on the surface of DO Cep get maximum energy in longer times, while the geometries of the flaring loops or areas get smaller.
\end{abstract}

Keywords: methods: data analysis — methods: statistical — stars: spots — stars: flare — stars: individual (DO Cep)

Received 2011 July 3, accepted 2011 September 26, published online 2011 November 7

\section{Introduction}

The white-light flares observed on the surfaces of UV Ceti-type stars and their process are not fully understood, although these subjects have been heavily studied (Benz and Güdel 2010). In this study, we obtained the largest data set from the observations of DO Cep in the literature. The data are very useful for a statistical analysis of the white-light flare property.

The observed star, DO Cep (Krüger 60B, KR 60B), is classified as a UV Ceti-type star in the SIMBAD database from spectral type dM4V (Henry et al. 1969). The star is a component of HD 239960, which is a visual binary (Lacy 1977; Söderhjelm 1999). The other component of the binary is GJ $860 \mathrm{~A}$, which is classified as dM3V by Henry et al. (1969); Tamazian et al. (1969). Unlike DO Cep, GJ $860 \mathrm{~A}(\mathrm{KR} 60 \mathrm{~A})$ is not active, as seen from the literature. In studies of the system, the semi-major axis of the orbit (a) was found to be 2.420 arcsec, while the orbital inclination $(i)$ was calculated as $172^{\circ}$ by Söderhjelm (1999). The orbital period was found to be 44.64 years, while the orbit eccentricity $(e)$ was computed as 0.41 in the same study. The distance of the system is given as $4.0 \mathrm{pc}$ by Pettersen (1991), while it is given as $4.04 \mathrm{pc}$ by Schmitt and Liefke (2004). Some basic properties taken from (Lacy 1977) are listed in Table 1 for each component.

According to Veeder (1974), DO Cep is an old disk star. In fact, taking $M_{b o l}=9.72 \mathrm{mag}$ and $\log \left(T_{\text {eff }}\right)=3.525$, its age was computed as about $5.0 \times 10^{8}$ years by Vandenberg et al. (1983). The equatorial rotational velocity $(v \sin i)$ of DO Cep was found to be $4.7 \mathrm{~km} \mathrm{~s}^{-1}$ by Glebocki and Gnacinski (2005); Jenkins et al. (2009). There are several flare patrols for DO Cep in the literature (Haro and Chavira 1955; Herr and Brcich 1969; Nicastro 1975; Contadakis et al. 1982). Haro and Chavira (1955) were the first to suspect that DO Cep is a flare star. Then, Herr and Brcich (1969) observed the star for 27.8 hours, detecting 10 flares. Nicastro (1975) detected 22 flares in 57.4 hours of flare patrol, while Contadakis et al. (1982) detected no flare in 59.13 hours of flare patrol. As seen from the literature, DO Cep has a high level of flare activity.

In this study, DO Cep was observed in the $U$-band for flare patrol in 2006 and 2007, and 89 white-light flares were detected. In order to classify the flares detected from DO Cep, the method described by Dal and Evren (2010) was used. In the literature, there are several studies about classifying white-light flares (Haro and Parsamian 1969; 
Table 1. Basic parameters for the components of visual binary Krüger 60 (all the parameters are taken from Lacy 1977)

\begin{tabular}{lll}
\hline Parameter & $\begin{array}{l}\text { KR 60A } \\
\text { (GJ 860 A) }\end{array}$ & $\begin{array}{l}\text { KR 60B } \\
\text { (DO Cep) }\end{array}$ \\
\hline$V(\mathrm{mag})$ & 9.850 & 11.220 \\
$V-R(\mathrm{mag})$ & 1.760 & 1.890 \\
$\pi \times 10^{3}(\operatorname{arcsec})$ & $251 \pm 5$ & $251 \pm 5$ \\
$\log \left(R_{\text {star }} / R_{\odot}\right)$ & $-0.45 \pm 0.05$ & $-0.65 \pm 0.05$ \\
$\log \left(M_{\text {star }} / M_{\odot}\right)$ & $-0.57 \pm 0.03$ & $0.80 \pm 0.03$ \\
\hline
\end{tabular}

Osawa et al. 1968; Moffett 1974; Gurzadian 1988). The classification of the flare light variations is important due to modelling these events (Gurzadian 1988; Gershberg 2005). The white-light flare events were generally classified into two subtypes as slow and fast flares (Haro and Parsamian 1969; Osawa et al. 1968). However, some studies, such as Oskanian (1969) and Moffett (1974), revealed that white-light flares can be classified into more than two subtypes. Kunkel (1967) revealed that the observed flare light-curves should be a combination of slow and fast flares. Recently, Dal and Evren (2010) developed a rule for classifying white-light flares. The rule depends on the ratio of flare decay times to flare rise times, and classifies a flare whose decay time is 3.5 times longer than its rise time as a fast flare. If the decay time of a flare is shorter than 3.5 times the length of its rise time, the flare can be classified as a slow flare. In fact, there are two possible energy sources for white-light flares (Gurzadian 1988). According to the author, thermal processes are dominant in the slow flare events, while nonthermal processes are dominant in the fast flare events. A rapid increase is generally seen in light curves if the energy source is caused by nonthermal processes (Benz and Güdel 2010; Gershberg 2005; Gurzadian 1988).

Apart from the shapes of the flare light variations, the upper and lower limits of both flare power and flare timescales are also important to understand the flare processes occurring on a star. In order to compare the flare powers of different stars, several studies have been done. In these studies, the flare energy spectra were derived for each star (Gershberg 1972; Lacy et al. 1976; Wall 1981; Gershberg and Shakhovskaya 1983; Pettersen et al. 1984; Mavridis and Avgoloupis 1986). According to the results of these studies, the energy levels of stars vary from one to the next. The variations seem to be caused by the different ages of stars. On the other hand, the analyses based on the flare energies may not give accurate results because the flare energy depends on the luminosity of a star as well as the power of the flare. Thus, if the stars are from different spectral types, the calculated energies of the flares will be different, even if their real powers are the same. In this respect, the flare equivalent duration was based on the analyses in this study in order to determine the behaviour of the white-light flares of DO Cep. The
Table 2. Basic parameters for DO Cep and its comparison (C1) and check (C2) stars

\begin{tabular}{lcc}
\hline Stars & $V(\mathrm{mag})$ & $B-V(\mathrm{mag})$ \\
\hline DO Cep & 9.615 & 1.604 \\
C1 = HD 239952 & 9.528 & 1.378 \\
C2 = SAO 34476 & 7.943 & 0.530 \\
\hline
\end{tabular}

method recently developed by Dal and Evren (2011a) is based on the modelling the distributions of the flare equivalent durations versus flare total durations. The authors demonstrate that the best function is the onephase exponential association function (hereafter the OPEA) to model the distribution. As is seen in the OPEA model, the flare equivalent durations cannot be higher than a specific value, and the flare's total duration does not matter. Dal and Evren (2011a) defined this level as an indicator for the saturation level for the white-light flare processes. In fact, white-light flares are detected in some large active regions, where compact and two-ribbon flares are occurring on the surface of the Sun (Rodonó 1990; Benz and Güdel 2010). It is possibly expected that the energies or the flare equivalent durations of white-light flares can also reach saturation. Generally, flare activity seen on the surfaces of dMe stars is modelled in terms of the processes of the solar flare event. This is why the magnetic reconnection process is accepted as the source of the energy in these events (Gershberg 2005; Hudson and Khan 1997). According to both some models and observations, it is seen that some parameters of magnetic activity can reach saturation (Gershberg 2005; Skumanich and McGregor 1986; Vilhu and Rucinski 1983; Vilhu et al. 1986; Doyle 1996a,b).

\section{Observations and Analyses}

\subsection{Observations}

The observations of the flare patrol were acquired with a high-speed three-channel photometer (HSTCP) attached to the 48-cm Cassegrain-type telescope at Ege University Observatory. Using a tracking star in the second channel of the photometer, flare observations were continued in the standard Johnson $U$-band with exposure times between 7 and $10 \mathrm{~s}$ and a time resolution of $0.01 \mathrm{~s}$. Considering the technical properties of the HSTCP given by Meištas (2002) and following the procedures outlined by Kirkup \& Frenkel (2006), the mean average of the standard deviations of observation times was computed as $0.08 \mathrm{~s}$ for the $U$-band observations. Some properties of DO Cep and its comparisons are listed in Table 2. Standard $V$ magnitudes and $B-V$ color indexes obtained in this study are given in Table 2. Although DO Cep and its comparison stars are very close to one another on the celestial plane, differential extinction corrections were applied. The extinction coefficients were obtained from observations of the comparison stars on each night. 
Moreover, the variable and its comparison stars were observed in the standard Johnson UBVR bands with the standard stars in their vicinity, and the reduced differential magnitudes, in the sense of variable minus comparison stars, were transformed to the standard system using the procedures described by Hardie (1962). The standard stars were chosen from the catalogues of Landolt (1992). Heliocentric corrections were applied to the times of the observations. The standard deviations of observation points acquired in the standard Johnson $U B V R$ bands were about $0.015 \mathrm{mag}, 0.009 \mathrm{mag}, 0.007 \mathrm{mag}$ and $0.007 \mathrm{mag}$ on each night, respectively. To compute the standard deviations of observations, we used the standard deviations of the reduced differential magnitudes in the sense comparisons $(\mathrm{C} 1)$ minus check $(\mathrm{C} 2)$ stars for each night. There is no variation in the standard brightness of the comparison stars. The flare patrol of DO Cep was continued for 9 nights between 2006 September 9 and 2006 November 26, and 11 nights between 2007 July 31 and 2007 October 17. The total duration of the $U$-band flare patrol was $22.76 \mathrm{~h}$ in 2006 and $44.85 \mathrm{~h}$ in 2007.4446 observing points were obtained in 2006, while 9841 observing points were obtained in 2007. According to the $3 \sigma$ of the $U$-band standard deviation in each night, it was decided whether an event observed in that night was a flare, or not. Therefore, 88 flares were detected in 2007, while only one flare could be detected in 2006.

Gershberg (1972) developed a method for calculating flare energies. Flare equivalent durations (s) and energies (erg) were calculated using Equations 1 and 2 of this method:

$$
P=\int\left[\left(I_{\text {flare }}-I_{0}\right) / I_{0}\right] d t
$$

where $I_{0}$ is the intensity of the star in the quiescent level and $I_{\text {flare }}$ is the intensity during the flare; and

$$
E=P \times L,
$$

where $E$ is the flare energy (erg), $P$ is the flare equivalent duration (s), and $L$ is the luminosity of the star in the quiescent level in the Johnson $U$-band.

The parameters of the flare light curves were calculated for each flare. All the parameters were computed following the procedure described in detail by Dal and Evren (2010). There are some important points in the procedure. We firstly separated each flare light curve into three parts. One of them is the part indicating the quiescent level of the brightness before the first flare on each night. The brightness level without any variations (such as a flare or any oscillation) was taken as the quiescent level of the brightness of this star. To determine this level, we used the standard deviation of each observation point, considering the mean average of all the observation points until this last point. If the standard deviations of that one and following points get over the $3 \sigma$ level, this point was taken as the beginning of a flare. Thus, the quiescent levels of each star were determined from all the observation points before the first flare on each night. We fitted this level with a linear function, and then, using this linear function, we computed the flare equivalent duration, flare amplitude and all the flare time scales (rise and decay times). The part of the light curve above the quiescent level was also separated into two sub-parts. The first of them is the impulsive phase, in which the flare increases, and the second is the decay phase. The impulsive and decay phases were separated according to the maximum brightness observed in this part. It must be noted that some flares have a few peaks. In this case, the point of the highest peak was assumed as the flare maximum. To determine the flare time scales, we fitted the impulsive and decay phases with polynomial functions. The best polynomial functions were chosen according to the correlation coefficients $\left(r^{2}\right)$ of fits. To determine the beginning and end of each flare, we computed the intersection points of the polynomial fits with the linear fit of the quiescent level and their standard deviations. In this study, the intersection points were taken as the beginning and end of each flare. The flare rise time was taken the duration between the beginning and the flare maximum point. In the same way, the flare decay time was taken the duration between the flare maximum point and the flare end. The height of the observed maximum point from the quiescent level was taken as the amplitude of the flare. The same procedure was used for each flare, and GRAPHPAD PRISM v5.02 (Motulsky 2007) software was used all calculations.

All calculated parameters are listed in Table 3 for the 89 flares. The observing date, heliocentric Julian date HJD of flare maximum time, flare rise and decay times (s), flare total duration (s), flare equivalent duration (s), flare amplitude in $U$-band (mag), $U-B$ color index (mag), flare energy (erg) and flare type are listed in the columns of the table. As is explained in Section 1, another important point is that the flare equivalent durations were used in the analyses due to the luminosity term $(L)$ in Equation 2, in stead of flare energies.

$$
N=\sum n_{f} / \sum T_{t}
$$

In addition to these parameters, following the method used by Leto et al. (1997) and Dal and Evren (2011b), the flare frequency $(N)$ was computed for each observing season. In Equation 3, $n_{f}$ is the total number of flares detected in a season, and $T_{t}$ is the total time of the flare patrol in that season. Using Equation 3, the value of $N$ was found to be 0.044 for 2006 and 3.866 for 2007 .

If the detected flares are examined, it will be seen that the light curve of each flare has a distinctive lightvariation shape. Five light-curve parts from the observations are seen in Figures 1-5, for example. The horizontal dashed lines seen in these figures represent the level of quiescent brightness. Three flares detected on 2007 August 1 are seen in Figure 1. According to the rule described by Dal and Evren (2010), the flares A and B 


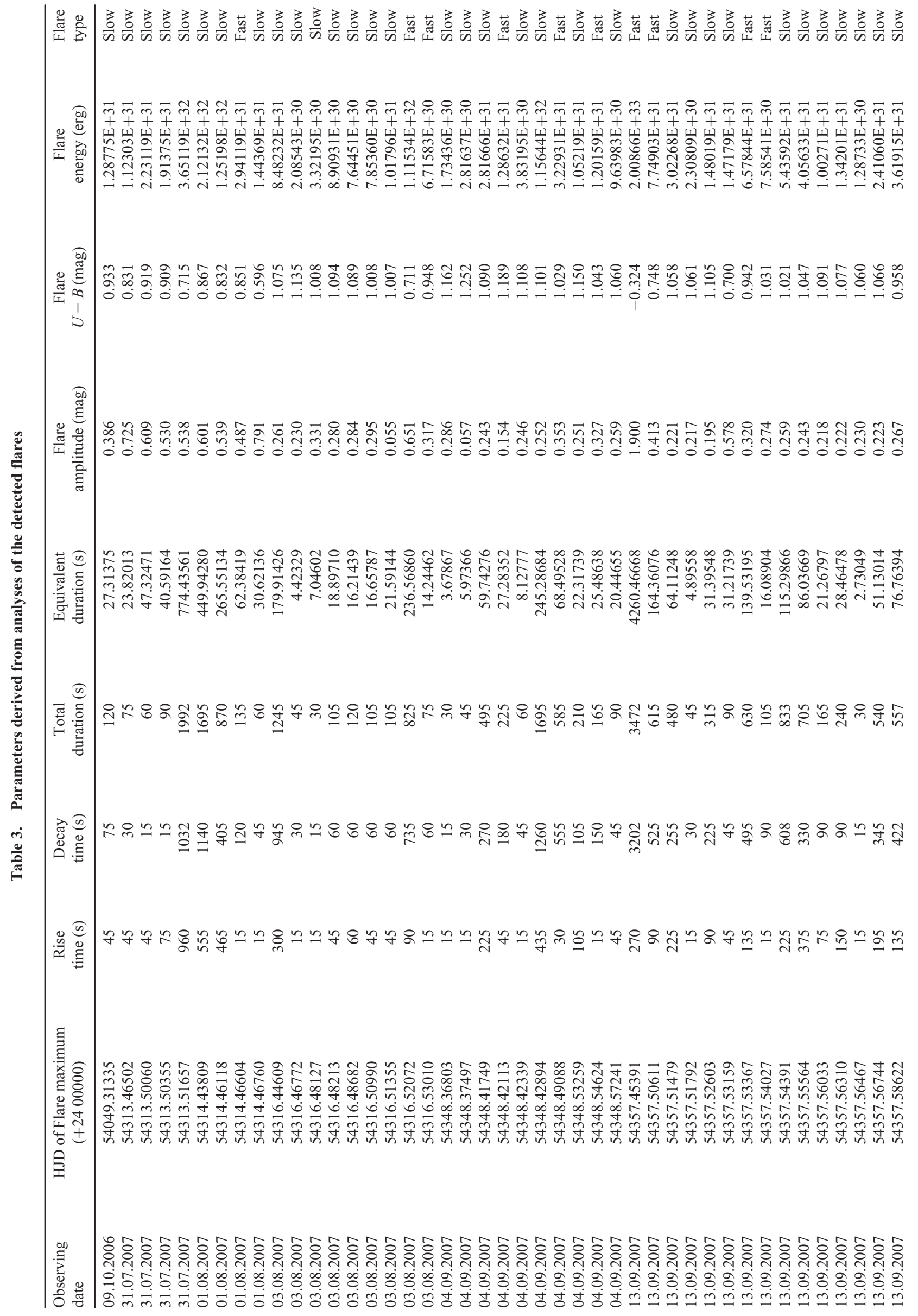




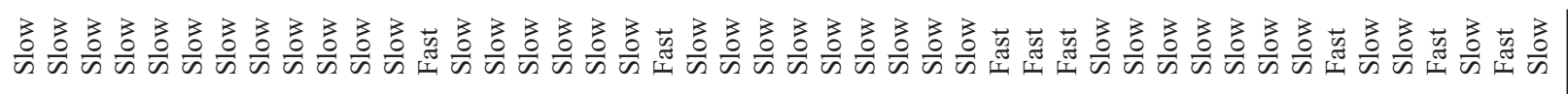

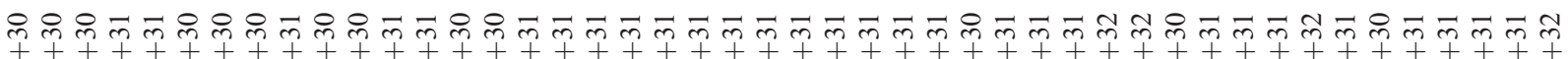

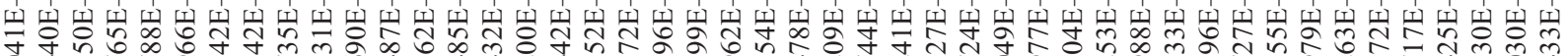

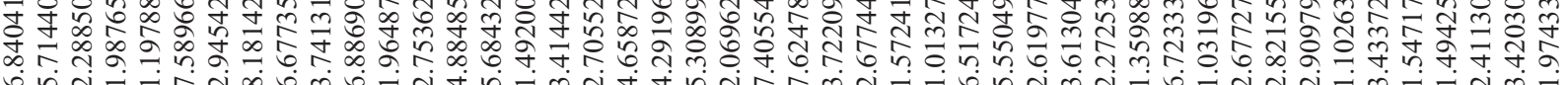

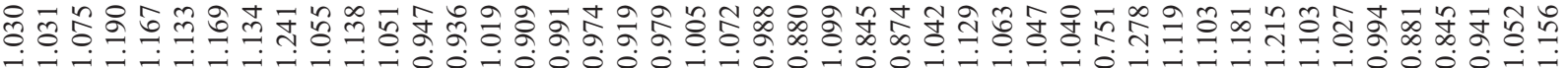

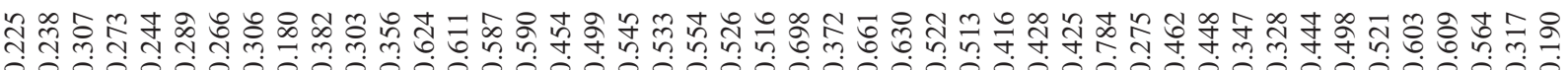

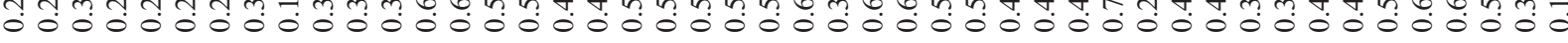

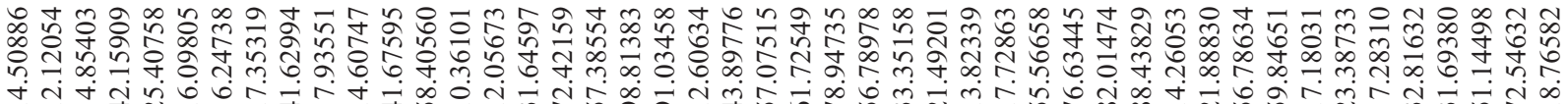

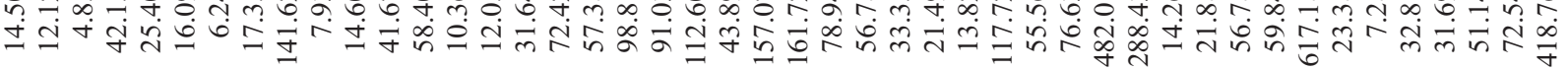

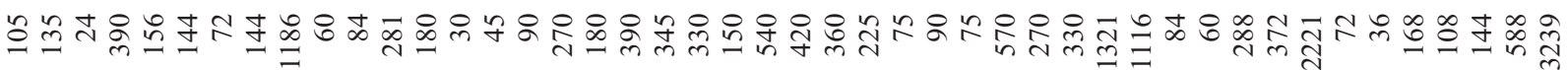

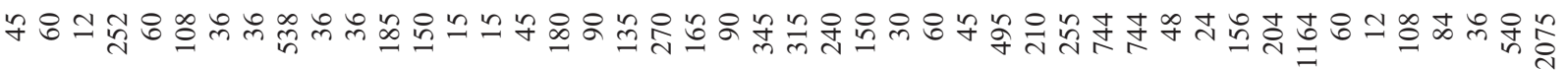

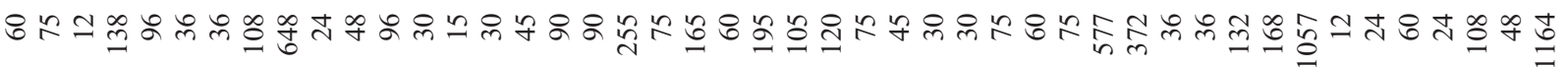

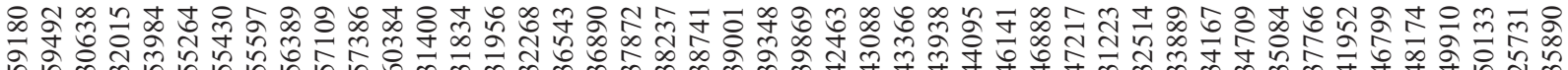

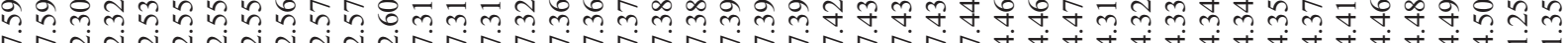

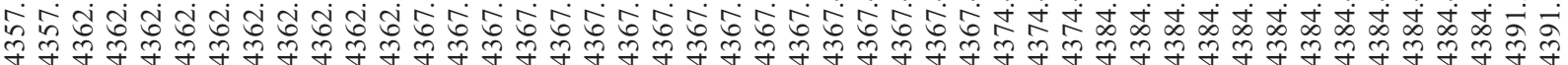

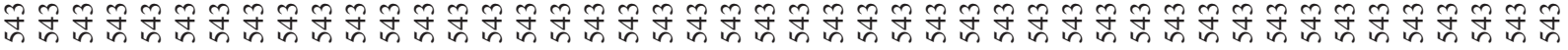

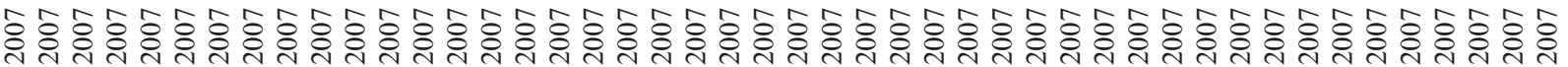

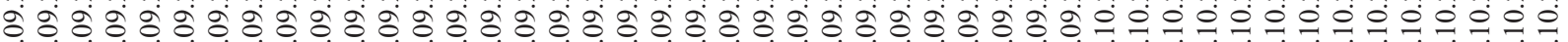

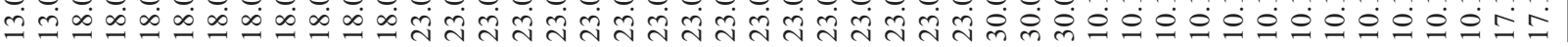




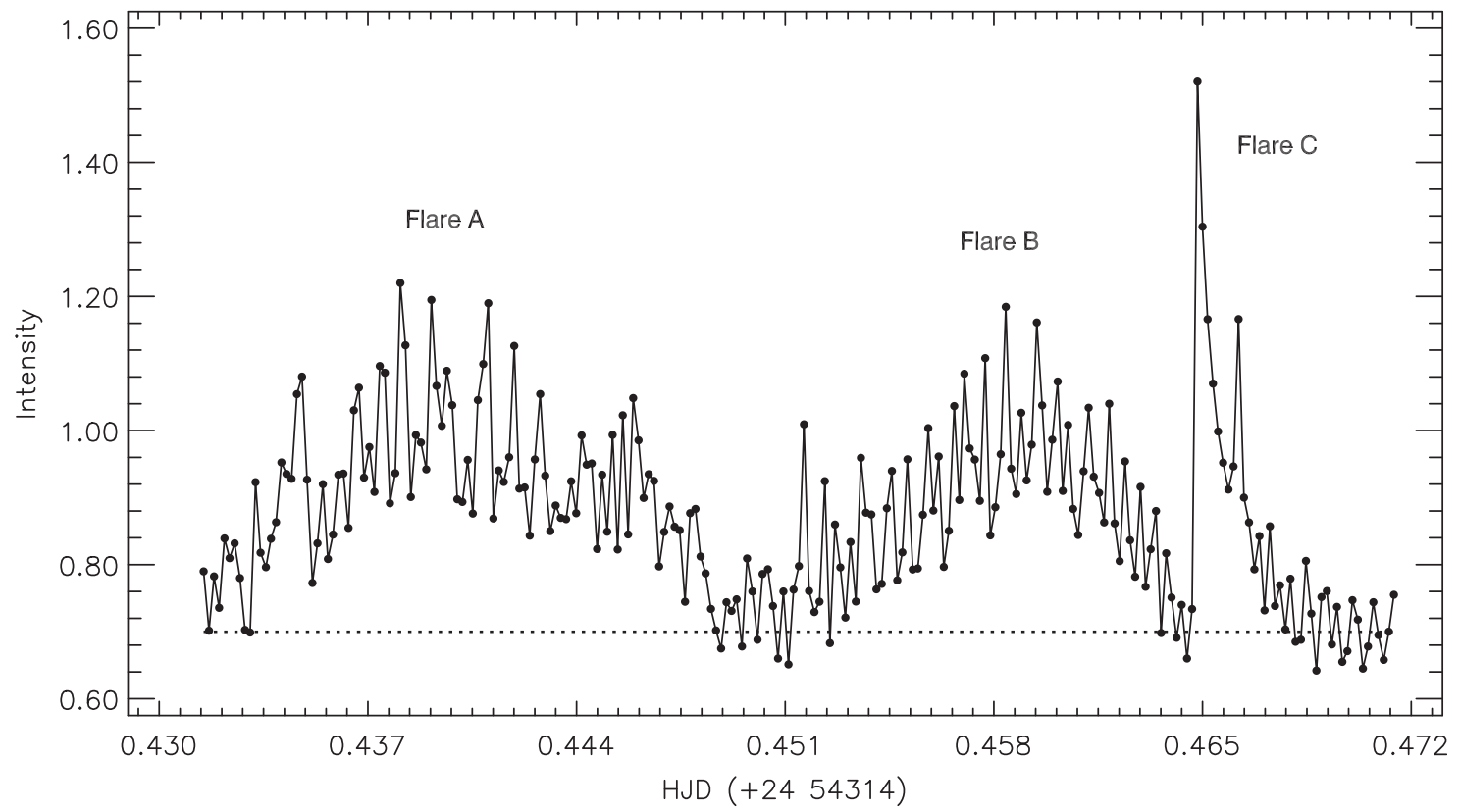

Figure 1 The flare light curves detected in $U$-band observations of DO Cep on 2007 August 1. Flares A and B are two examples of slow flares, while flare $\mathrm{C}$ is an example of a fast flare.

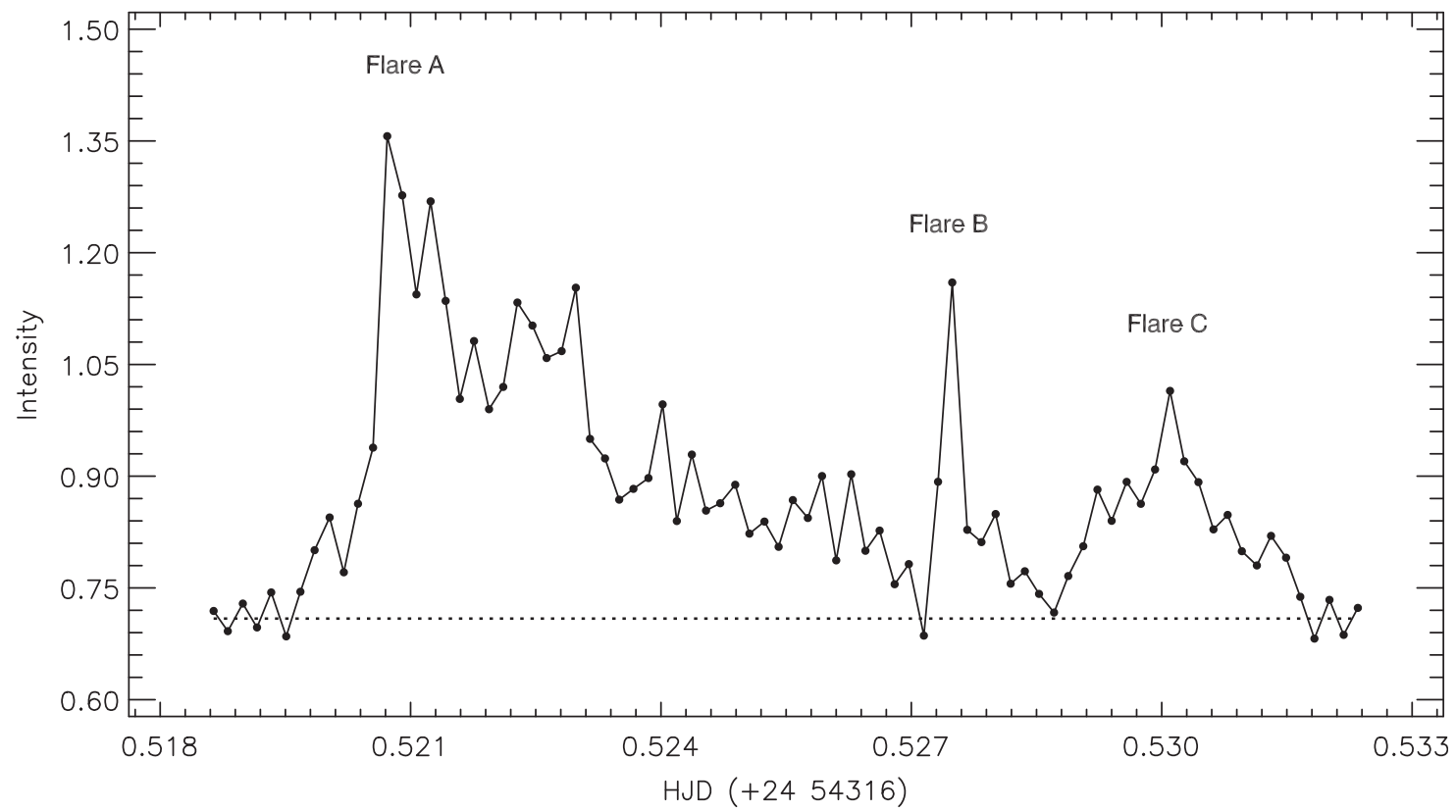

Figure 2 The flare light curves detected in $U$-band observations of DO Cep on 2007 August 3. Flares A and B are two examples of slow flares, while flare $\mathrm{C}$ is an example of a fast flare.

shown in Figure 1 are two slow flare examples, while flare $\mathrm{C}$ is an example of a fast flare. The flares seen in Figure 2 were detected on 2007 August 3. In this figure, flares $\mathrm{A}$ and $\mathrm{B}$ are examples of fast flares, while flare $\mathrm{C}$ is a slow flare. Figure 3 shows two flares, and both of them are fast flares. Flare A in this figure is the most powerful flare detected in the study. Its amplitude is $1.90 \mathrm{mag}$ in the $U$-band. Its rise time is $270 \mathrm{~s}$, and decay time is $3202 \mathrm{~s}$. The flare seen in Figure 4 is a combined flare. There should actually be two flares (part A and part B), but their light variations are combined in the light curves. Moffett (1974) classified flares like this as a complex flares. It should be noted that flares like this one are not ignored in the analyses described in Section 2.2. More interesting examples are seen in Figure 5. In the figure, flares A and B are slow flares, while flare $\mathrm{C}$ is a fast flare. Apart from these three flares, there are three spikes, which are combined with flares A and B. 


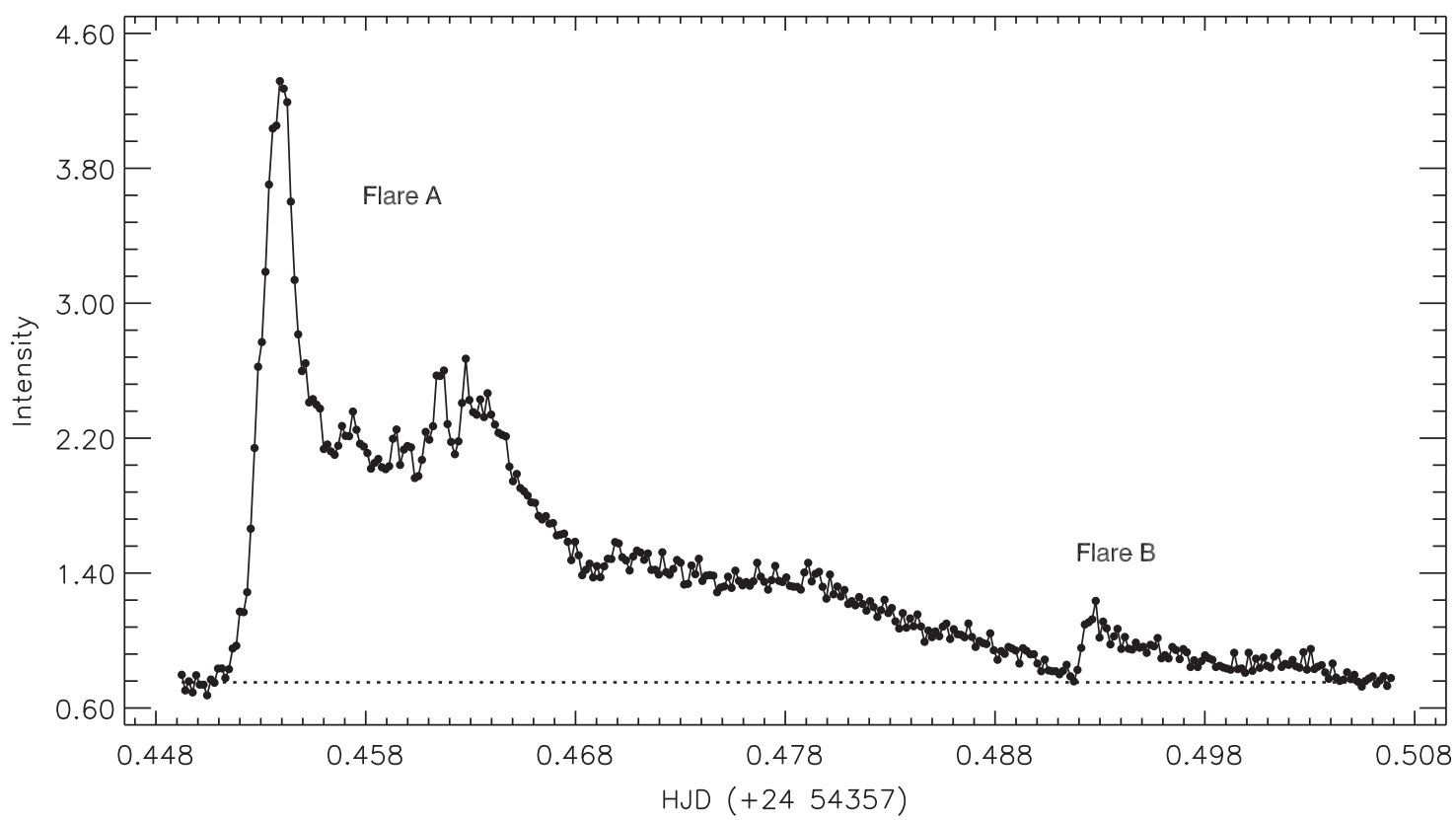

Figure 3 The flare light curves detected in $U$-band observations of DO Cep on 2007 September 13. Flares A and B are two examples of slow flares.

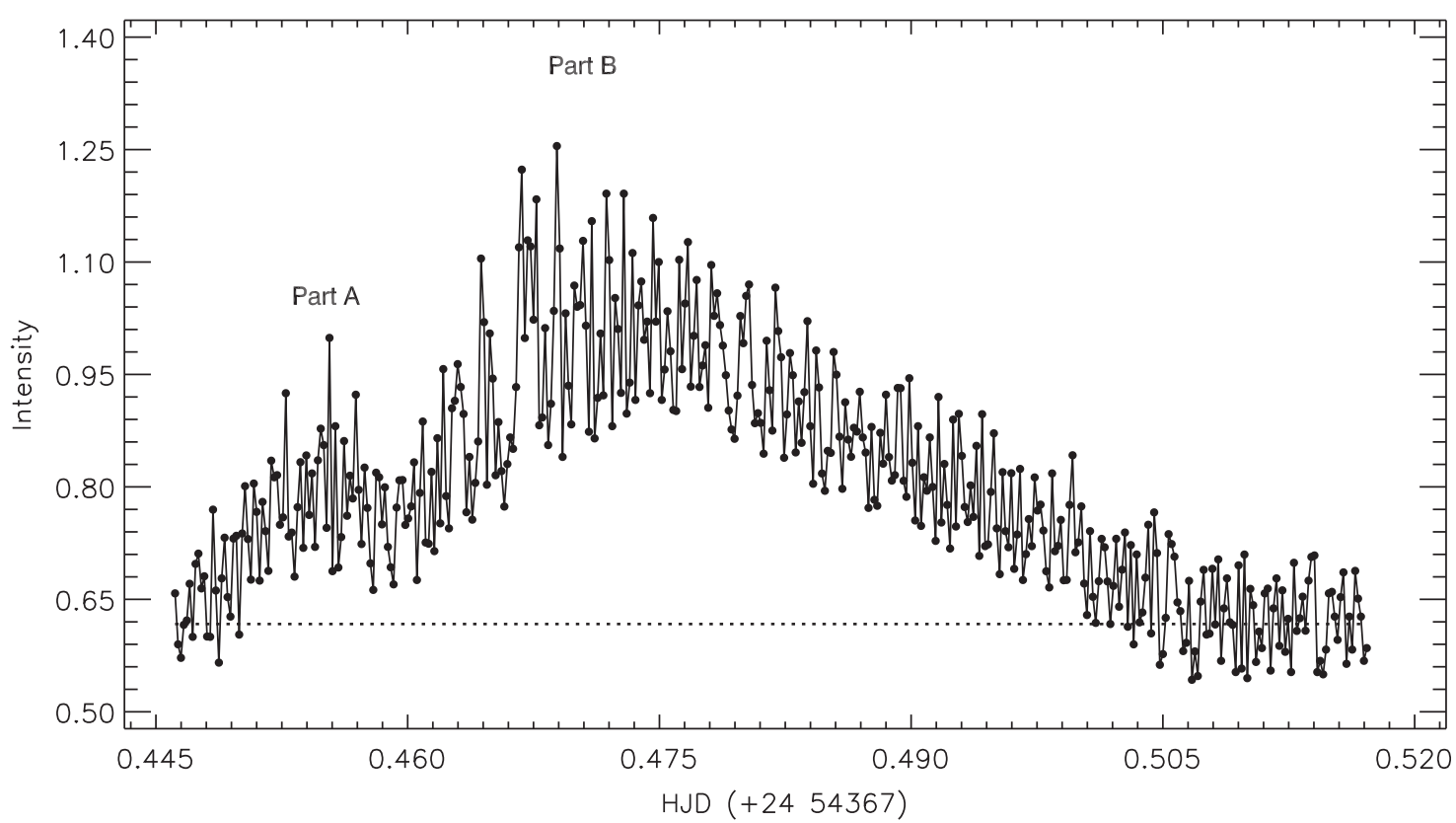

Figure 4 A flare light curve detected in $U$-band observations of DO Cep on 2007 September 23. This flare light curve is an example of a combined flare. Part A is part of one flare, while part B is a part of another. Both component flares would probably be slow flares.

\subsection{Fast and Slow Flares}

The flares detected from DO Cep were analysed using the method developed by Dal and Evren (2010). It was tested whether the limit ratio (3.50) is also acceptable for the white-light flares detected from DO Cep. Thus, using a new large data set, it was tested whether or not the value of 3.50 is a general limit.

In the first step, the equivalent durations of flares with equal rise times were compared. For example, there are 13 flares with a rise time of $15 \mathrm{~s}$. The light variations of
9 of these 13 flares are similar to flares A and B seen in Figure 1. The light variations of the other 4 flares are similar to flare $\mathrm{C}$ shown in Figure 1. The average of equivalent duration for the 9 flares similar to flares $\mathrm{A}$ and $\mathrm{B}$ is $6.206 \mathrm{~s}$. However, the average equivalent duration for the other 4 flares is $29.551 \mathrm{~s}$. The main difference between these two example groups is seen in the shapes of the light curves. Finally, we found 18 flares with higher energy and 71 flares with lower energy among the 83 flares detected from DO Cep. 


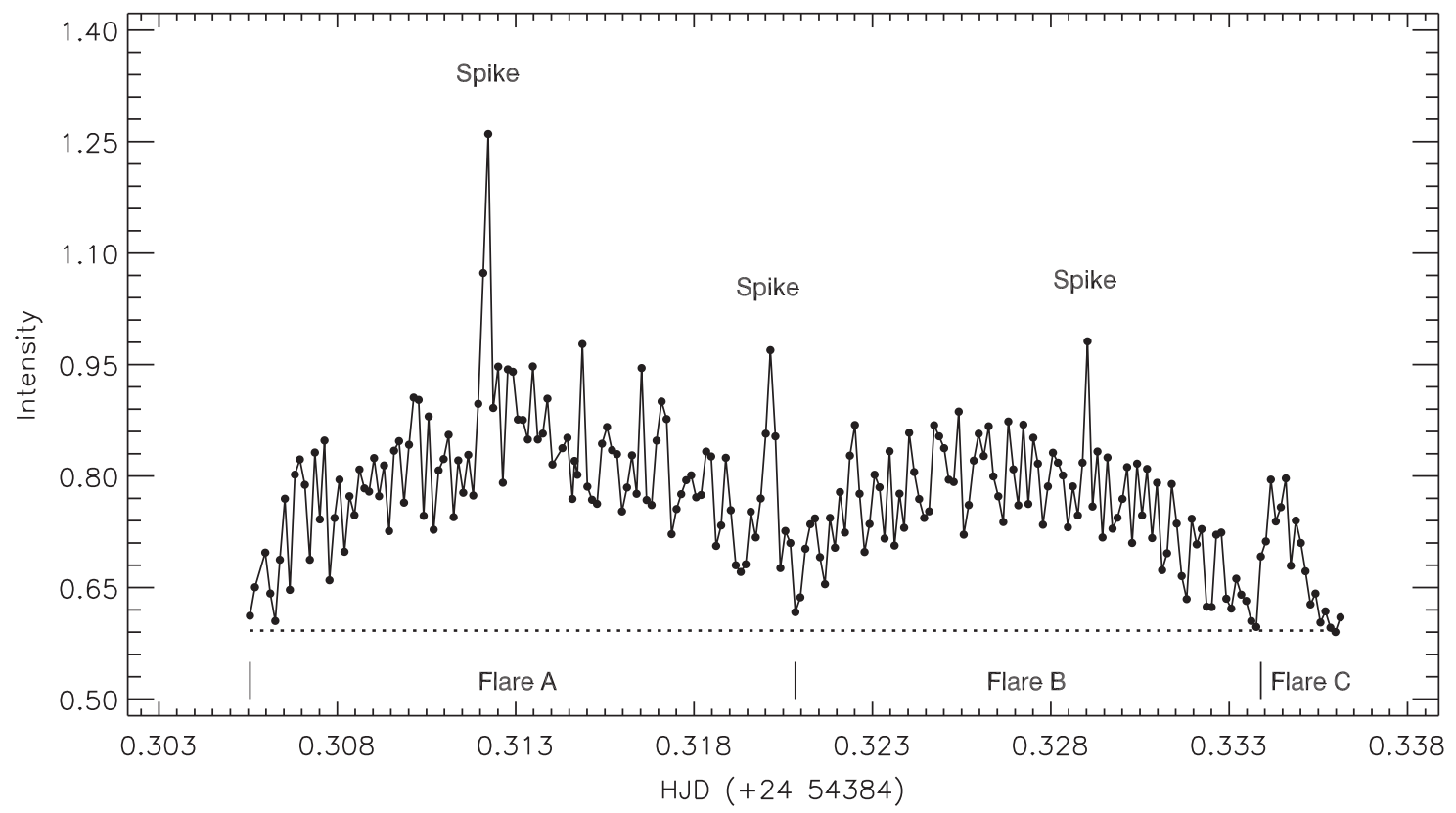

Figure 5 The flare light curves detected in $U$-band observations of DO Cep on 2007 October 10. All three labelled flares are examples of slow flares. Additionally, three impulsive spikes are seen during the first two slow flares.

Table 4. The results obtained from both the regression calculations and the $t$-test analyses performed to the mean averages of the equivalent durations $\left(\log P_{u}\right)$ versus flare rise times $\left(\log T_{r}\right)$ in the logarithmic scale are listed for both fast and slow flares whose rise times are the same

\begin{tabular}{|c|c|c|}
\hline Flare groups & Slow flare & Fast flare \\
\hline \multicolumn{3}{|l|}{ Best representation values } \\
\hline Slope & $1.046 \pm 0.048$ & $1.232 \pm 0.181$ \\
\hline$y$-intercept when $x=0.0$ & $-0.450 \pm 0.095$ & $-0.137 \pm 0.302$ \\
\hline$x$-intercept when $y=0.0$ & 0.430 & 0.111 \\
\hline \multicolumn{3}{|l|}{ Mean average of all $y$ values } \\
\hline Mean average & 1.544 & 1.871 \\
\hline Mean average error & 0.067 & 0.130 \\
\hline \multicolumn{3}{|l|}{ Goodness of fit } \\
\hline$r^{2}$ & 0.871 & 0.744 \\
\hline \multicolumn{3}{|c|}{ Is slope significantly non-zero? } \\
\hline$p$-value & $<0.0001$ & $<0.0001$ \\
\hline Deviation from zero? & Significant & Significant \\
\hline
\end{tabular}

Using the independent samples $t$-test (hereafter simply $t$-test) (Wall and Jenkins 2003; Dawson and Trapp 2004) in SPSS v17.0 (Green et al. 1999) and GRAPHPAD PRISM v5.02 (Motulsky 2007) software, data sets were analysed in order to test whether these two groups are statistically independent of each other. In the analyses, the flare rise times were taken as a dependent variable, and the flare equivalent durations were taken as an independent variable. The value of $\alpha$ is taken as 0.005 , which allowed us to test the statisical acceptability of the results (Dawson and Trapp 2004).

The mean average of the equivalent durations for 71 slow flares was found to be $1.544 \pm 0.067 \mathrm{~s}$, and it was computed as $1.871 \pm 0.130 \mathrm{~s}$ for the 18 fast flares in the logarithmic scale. This shows that there is a difference of about $0.327 \mathrm{~s}$ between average equivalent durations in the logarithmic scale. The probability value (hereafter $p$-value) was computed to test the results of the $t$-test, and it was found to be $p<0.0001$. Considering the $\alpha$ value, this means that the result is statistically acceptable. All the results obtained from the $t$-test analyses are given in Table 4.

In the second step, the distributions of the equivalent durations $\left(\log P_{u}\right)$ versus flare rise times $\left(\log T_{r}\right)$ were modelled for both flare types. Using the least-squares method, the best models for the distributions were examined in SPSS V17.0 and GRAPHPAD PRISM v5.02 software. The regression calculations demonstrated that the best fits of the distributions are linear functions. The derived linear fits given by Equations 4 and 5 are shown in Figure 6.

$$
\begin{aligned}
& \log P_{u}=1.232 \times \log T_{r}-0.137 \\
& \log P_{u}=1.046 \times \log T_{r}-0.450
\end{aligned}
$$




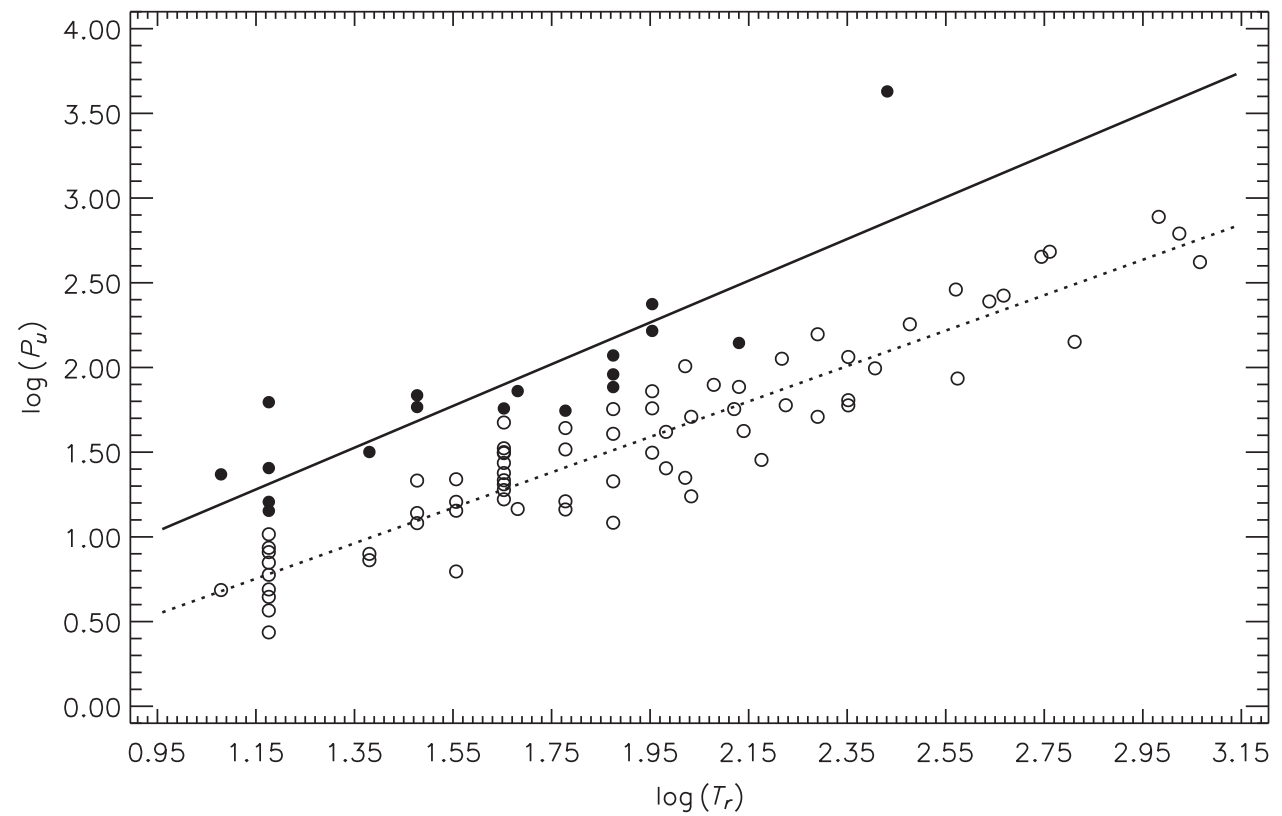

Figure 6 Distributions for the mean averages of the equivalent durations $\left(\log P_{u}\right)$ vs. flare rise times $\left(\log T_{r}\right)$ in the logarithmic scale. Open circles represent slow flares, while filled circles represent fast flares. Lines represent fits given in Equations 4 and 5.

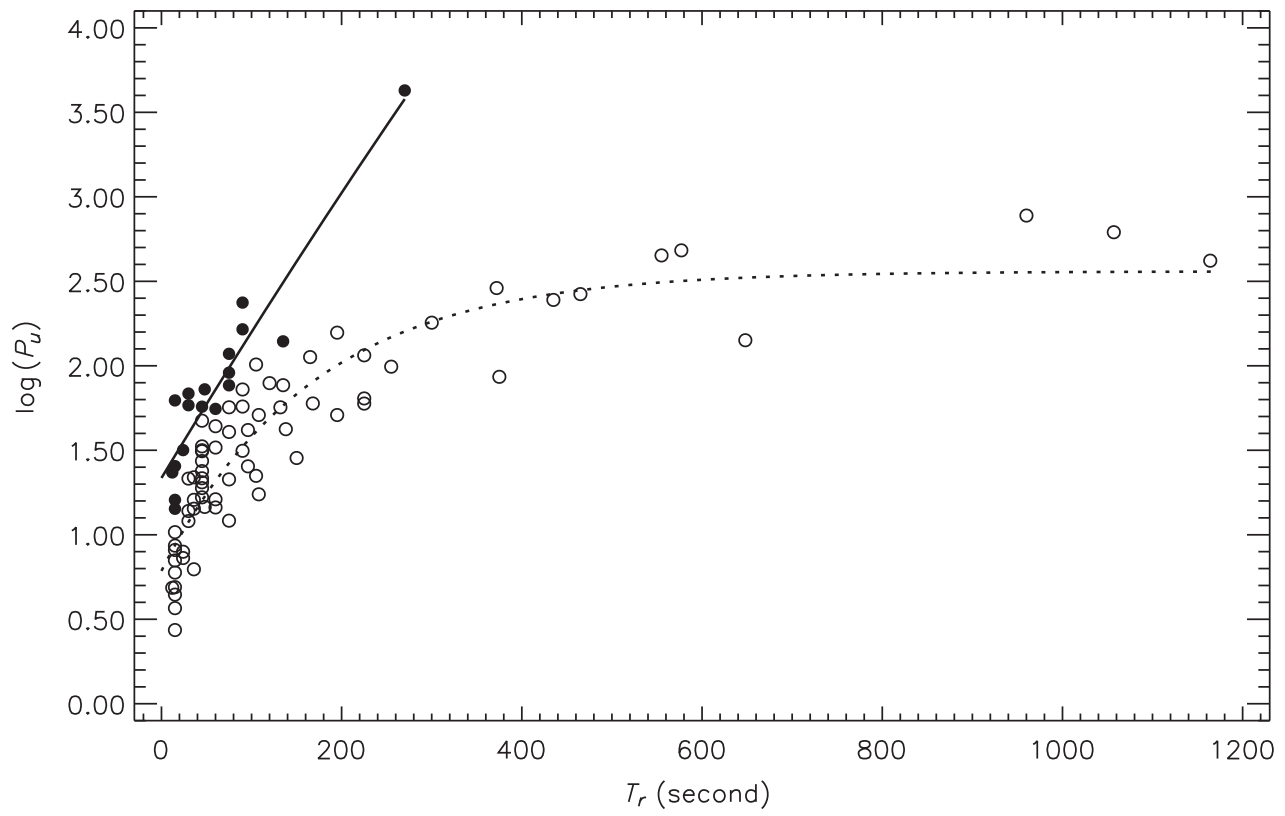

Figure 7 Distributions of the equivalent durations $\left(\log P_{u}\right)$ in the logarithmic scale vs. flare rise times $\left(T_{r}\right)$ for all 89 flares detected in observations of program stars. Open circles represent slow flares, while filled circles represent fast flares.

In the next step, the linear functions were compared. The slope of the linear function was found to be $1.046 \pm 0.048$ for slow flares, while it was computed as $1.232 \pm 0.181$ for fast flares. The $p$-value was calculated and found to be $p=0.650$. The $p$-value indicates that there is no significant difference between the slopes of fits, and it can be assumed that they are statistically parallel.

Finally, the $y$-intercept values of both linear fits were compared. The $y$-intercept value was found to be -0.450 for the slow flares, and it was found to be -0.137 for the fast flares in the logarithmic scale. There is a difference of about 0.313 between them. Then, the $p$-value was computed for the $y$-intercept values to determine whether there is a statistically significant difference; it was found to be $p<0.0001$. The result demonstrated that the difference between the two $y$-intercept values is obviously important.

The distributions of the equivalent durations in the logarithmic scale versus flare rise times were modelled with the OPEA function to find the maximum energy levels and timescales of the two flare types. The derived distributions are shown in Figure 7. Using the leastsquares method, the regression calculations showed that the average value of the upper limit is $2.559 \pm 0.096$ for 


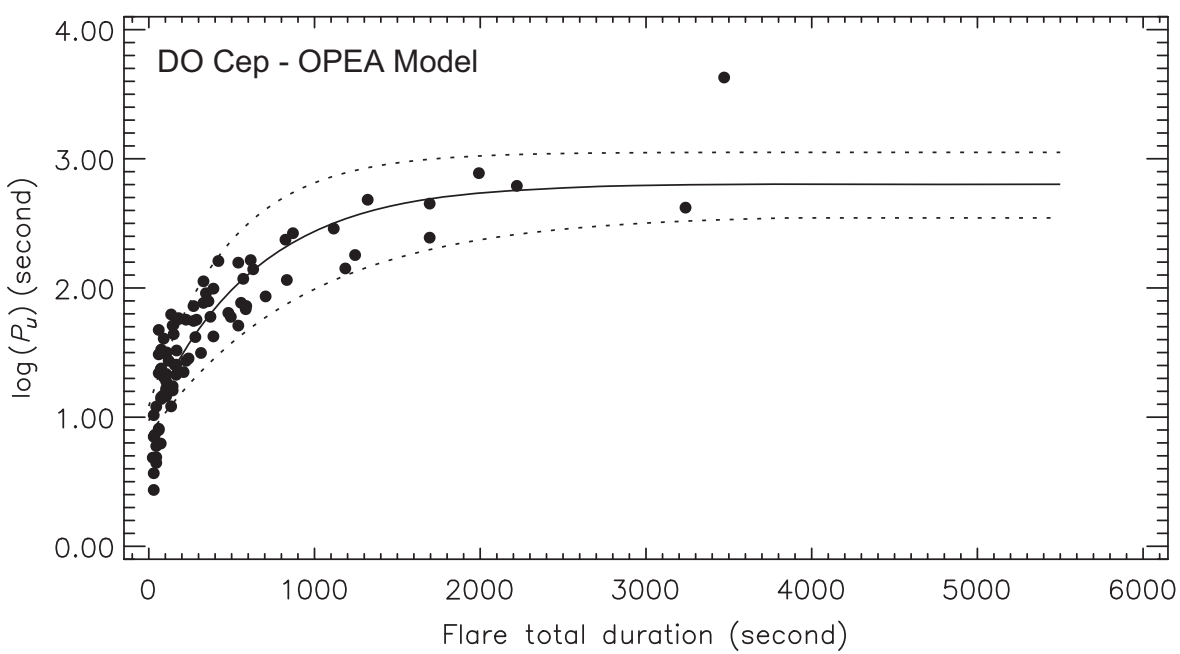

Figure 8 Distributions of flare equivalent duration on a logarithmic scale vs. flare total duration. Filled circles represent equivalent durations computed from flares detected from DO Cep. The line represents the model identified with Equation 6 computed using the least-squares method. The dotted lines represent $95 \%$ confidence intervals for the model.

Table 5. Using the least-squares method, the parameters were obtained from the OPEA function

\begin{tabular}{lcc}
\hline Parameter & Value & Error \\
\hline$y_{0}$ & 0.972 & 0.057 \\
Plateau & 2.810 & 0.057 \\
$k$ & 0.001601 & 0.000258 \\
$\tau$ & 624.8 & - \\
Half-life & 433.1 & - \\
Span & 1.837 & 0.115 \\
\hline
\end{tabular}

the slow flares. On the other hand, the regression calculations indicate that the distribution cannot be modelled with the OPEA function due to the linear increase. The linear fit derived for the fast flares is also seen in Figure 7. Besides the averaged value of the upper limit, it was found that the lengths of rise times for slow flares can reach $1164 \mathrm{~s}$, while they are not longer than $270 \mathrm{~s}$ for fast flares.

\subsection{The One-Phase Exponential Association Models of the Distribution of the Flares}

The distribution of the flare equivalent durations in the logarithmic scale versus the flare total durations indicates that the flare mechanism occurring on the surface of DO Cep has a upper limit for the flare energy. To examine this case, the distribution was modelled and statistically analysed. First of all, the distribution of the equivalent durations $\left(\log P_{u}\right)$ in the logarithmic scale versus the flare total durations was obtained. Then, using regression calculations, the best function was determined to fit the distribution by SPSS v17.0 software. The regression analyses demonstrated that the OPEA function (Motulsky 2007; Spanier \& Oldham 1987) given by Equation 6 is the best model fit. According to Dal and Evren (2011a), this is actually an expected case. The case demonstrates that the flares occurring on the surface of DO Cep have an upper limit for producing energy. In the final step, the OPEA model of the distributions was derived with GRAPHPAD PRISM v5.02 using the least-squares method.

$$
y=y_{0}+\left(\text { plateau }-y_{0}\right) \times\left(1-e^{-k x}\right)
$$

The details of the OPEA function have been given by Dal and Evren (2011a). In brief, some important parameters can be derived from the OPEA function, and these parameters reveal the condition of the flare mechanism occurring on the surface of the star. One of them is $y_{0}$, which is the lower limit of equivalent durations for observed flares in the logarithmic scale. In contrast to $y_{0}$, the parameter of plateau is the upper limit. It should be noted that the $y_{0}$ parameter depends on the quality of observations as well as flare power. However, the plateau parameter depends only on the power of flares. Dal and Evren (2011a) identified the plateau parameter as a saturation level for the white-light flare activity observed in the $U$-band. The derived OPEA model is shown in Figure 8 , while the parameters of the model are listed in Table 5. The span value listed in the table is the difference between the values of plateau and $y_{0}$. One of the most important parameters is the half-life value. This parameter is equal to half of the first $x$ value where the model reaches the plateau value. In other words, it is half of the flare's total duration, where flares with the highest energy start to be seen. In order to test the the plateau values derived from the OPEA model, the upper limit of the equivalent durations was computed using the $t$-test. Thus, the statistical acceptability of the plateau value was tested. The flares in the plateau phases of the model were only used to test. The mean average of the equivalent durations was computed and found to be $2.808 \pm 1.149$.

As seen from the data distributions, the maximum flare rise time obtained from these 89 flares is $1164 \mathrm{~s}$, while the maximum flare total duration is $3472 \mathrm{~s}$. 


\section{Results and Discussion}

\subsection{Flare Activity and Flare Types}

In this study, 89 white-light flares were detected in $U$-band observations of DO Cep. 88 flares were detected in the 44.85-h flare patrol of 2007, while only one flare was detected in the 22.76-h flare patrol of 2006. Therefore, 0.044 flares were detected per hour in 2006, while 3.866 flares were detected per hour in 2007 . There is a large difference between the flare frequencies $\left(N_{2006}\right.$ and $N_{2007}$ ) of consecutive observing seasons. Large differences between the flare frequencies obtained in different years are also seen in the literature (Herr and Brcich 1969; Nicastro 1975; Contadakis et al. 1982). According to the results of Herr and Brcich (1969), the flare frequency $(N)$ was 0.360 in $1968 . N$ was 0.509 flares per hour in 1970 , and it was 0.208 flares per hour in the observing season of 1972-1973 (Nicastro 1975). However, Contadakis et al. (1982) detected no flares in 1975. Consequently, the largest frequency was obtained with 3.866 flares per hour in this study. It seems that DO Cep is active as well as EV Lac, EQ Peg and AD Leo (Moett 1974; Dal and Evren 2010). The flare frequency variation of UV Ceti type stars has been examined in several studies (Ishida et al. 1991; Leto et al. 1997). Ishida et al. (1991) found no variation for a few stars, while Leto et al. (1997) demonstrated that the flare frequency of EV Lac is dramatically increasing. It must be noted that DO Cep requires a lengthier observing program, because its flare frequency varies a great deal.

The flares detected in this study are examined one by one. The flares, whose rise times are equal, were determined. It was seen that the flares are accumulating into two groups. It was seen that even if the rise times of two flares are equal, their equivalent durations can be different from each other. Apart from their equivalent durations, the main difference between two type flares is lightvariation shapes. As seen from Figures 1-5, some flares slowly increase and slowly decrease, while some of them increase rapidly but decrease slowly.

In logarithmic scale, the flare distributions were obtained for both groups. First of all, two group flares were analysed with the $t$-test. Then, they were modelled with the linear function, and the models of two groups were analysed for comparison. Using the $t$-test, the averages of equivalent durations for two types of flares were computed. The average of equivalent durations was found to be $1.871 \mathrm{~s}$ for the fast flares and $1.544 \mathrm{~s}$ for the slow flares. The difference of $0.327 \mathrm{~s}$ between these values in the logarithmic scale is equal to the $39.282 \mathrm{~s}$ difference between the equivalent durations. As can be seen from Equation 2, this difference between average equivalent durations affects the energies in the same way. Therefore, there is a difference of 39.282 times between the energies of these two types of flares. This difference must be the difference mentioned by Gurzadian (1988). On the other hand, according to Dal and Evren (2010), the difference between the two flare types is about 157 times.
In the case of DO Cep, the energies of the slow and fast flares occurring on the surface of the star seem to be closer to each other.

Apart from the average of equivalent durations, the parameters of the linear fits were also compared. The slope of the linear fit is 1.046 for the slow flares, which are low-energy flares, and it is 1.232 for the fast flares, which are high-energy flares. According to the $p$-value, the slopes are almost close to each other. It demonstrates that the flare equivalent durations versus the flare rise times increase in similar ways for both groups. However, the fast flare (flare A) seen in Figure 3 is seen out of the general trend of the fast flares. This flare is the most powerful flare detected in the study. It seems to be an extreme example. In the case of extreme examples, some effects must be involved in the fast flare process causing the long rise times. These effects can make fast flares seem more powerful than they actually are. However, comparing the $y$-intercept values of the linear fits, it is seen that there is a 0.313 times difference in the logarithmic scale. There is a 0.327 times difference between the general averages. Both values are close to each other. It means that the energy emitting-processes behave similarly, except the extreme flare. Apart from the equivalent durations, the differences between these two flare types are seen in the lengths of their rise times and their amplitudes. The maximum rise time seen among the slow flares is $1164 \mathrm{~s}$, but it is $270 \mathrm{~s}$ for fast flares. In addition, the amplitudes of slow flares can reach to $0.791 \mathrm{mag}$ at most, while the amplitudes of fast flares can reach to $1.900 \mathrm{mag}$.

Dal and Evren (2010) computed the ratios of flare decay times to flare rise times for two types of flares. They have demonstrated that there is a limit value between the two flare types. This limit value of the ratio of flare decay time to flare rise time is 3.50 . In this study, the limit value of this ratio is found to be 3.40 for the flares detected from DO Cep. Providing this limit value between flare types, it was found that the fast flare rate describes $20.22 \%$ of the 89 flares observed in this study, while the slow flare rate describes $79.78 \%$. It means that one of every five flares is a fast flare, the other four are slow flares. This result is close to what Gurzadian (1988) stated. According to Gurzadian (1988), slow flares with low energies and low amplitudes make up $95 \%$ of all flares, and the remainder are fast flares.

\subsection{Saturation Levels in the Detected White-Light Flare}

The distributions of flare equivalent durations versus flare total duration were modelled by the OPEA function expressed by Equation 6 for 89 white-light flares detected in observations of DO Cep. To model the distribution, the best model curve was sought. Considering the $p$-value and the correlation coefficient $\left(r^{2}\right)$ parameters, the OPEA function was found to be the best model function. The main characteristic feature of the OPEA is that this 
function has a plateau phase. According to the observations, the flare equivalent durations increase with the flare total duration until a specific total duration value. After the specific total duration, the flare equivalent durations are constant, and the total duration does not matter. There is just one flare among all of them. This flare is flare A seen in Figure 3. It must be an extreme sample.

Some parameters, such as plateau value, half-life and so on, were derived from the OPEA model. The plateau value was found to be 2.810 . The value is in agreement with the mean average of the equivalent durations. It had been found to be 2.808 . Considering the standard deviations of two values, they can be assumed to be equal. Besides, the found plateau value is also in agreement with the plateau values found from other stars by Dal and Evren (2011a). According to the $B-V$ colour index of DO Cep, it is seen that the star is among its analogues. This result supports that the upper limit of the energy producing the white-light flare mechanism increases towards the later spectral types.

It is well known that white-light flares occur in the regions where the compact and two-ribbon flare events are seen (Rodonó 1990; Benz and Güdel 2010). In the analyses, the flare-equivalent durations were used instead of the flare energies. In fact, the derived plateau values depend only on the power of the white-light flares. According to observations, the plateau phase exists in the model. The flare equivalent durations cannot be higher than a particular value, and the flare's total duration does not matter. Apart from the timescales, the power of the flares must depend on some other parameters, such as magnetic field flux and/or particle density in the volume of the flare processes. However, Doyle (1996a) and Doyle (1996b) suggested that the saturation in the active stars does not have to be related to the filling factor of magnetic structures on the stellar surfaces or the dynamo mechanism under the surface. It can be related to some radiative losses in the chromosphere, where the temperature and density are increasing in the case of fast rotation. This phenomenon can occur in the chromosphere due to the flare process instead of fast rotation, and this causes the plateau phase to occur in the distributions of flare equivalent duration versus flare total duration. On the other hand, the plateau phase cannot be due to some radiative losses in the chromosphere with increasing temperature and density. This is because Grinin (1983) demonstrated the effects of radiative losses in the chromosphere on the white-light photometry of the flares. According to Grinin (1983), the negative H opacity in the chromosphere causes the radiative losses, and these are seen as a pre-flare dip in the light curves of the white-light flares. Unfortunately, considering the results of Dal and Evren (2011a), it is seen that the plateau values vary from one star to the next. This indicates that some parameters, or their efficacies, which make the plateau increase, are changing from star to star. According to the standard magnetic reconnection model developed by Petschek (1964), there are several important parameters giving shape to flare events, such as Alfvén velocity $\left(v_{A}\right), B$, the emissivity of the plasma $(R)$ and the most important one, the electron density of the plasma $\left(n_{e}\right)$ (Van Den Oord and Barstow 1988; Van Den Oord et al. 1988). All these parameters are related to both heating and cooling processes in a flare event. Van Den Oord et al. (1988); Van Den Oord and Barstow (1988) have defined the radiative loss timescale $\left(\tau_{d}\right)$ as $E_{\mathrm{th}} / R$. Here $E_{\mathrm{th}}$ is the total thermal energy, while $R$ is the emissivity of the plasma. $E_{\text {th }}$ depends on the magnetic energy, which is defined as $B^{2} / 8 \pi$, and $R$ depends on the electron density $\left(n_{e}\right)$ of the plasma. $\tau_{d}$ is firmly correlated with $B$ and $n_{e}$, while $\tau_{r}$ is proportional to a larger loop length $(l)$ and smaller $B$ values. Consequently, it is seen that both the shape and power of a flare event depend mainly on two parameters, $n_{e}$ and $B$.

As is seen from the OPEA model, the flare equivalent durations start to reach maximum values in a specific total duration, and the half-life value was found to be $433.1 \mathrm{~s}$ from the model. In addition, the maximum flare rise time was found to be $1164 \mathrm{~s}$, while the maximum flare total duration was found to be 3472 s. These results demonstrated that the timescales of the flares detected from DO Cep are in fact shorter than they are in the earlier spectral types. However, the flares reach the maximum energy limits in longer times. It is well known from X-ray observations of the flares that the timescales of the $\mathrm{X}$-ray flares give some clues about the flaring loop geometry on the stars (Reeves and Warren 2002; Imanishi et al. 2003; Favata et al. 2005; Pandey and Singh 2008). White-light flares can exhibit the same behaviour as their X-ray counterparts (Gershberg 2005; Benz and Güdel 2010). If this case is valid, the timescales derived from the white-light flares can also give some clues about the flaring loop geometry or the flaring area geometry (at least for the photosphere). The obtained timescales from the observations of DO Cep demonstrated that the flaring loop or area is smaller than those seen on the stars from the earlier spectral types, because the obtained maximum flare duration for DO Cep flares is $3472 \mathrm{~s}$. The observed maximum durations are $5236 \mathrm{~s}$ for V1005 Ori and 4164 s for AD Leo (Dal and Evren 2011a). The flare timescales of both stars are dramatically longer than that of DO Cep. However, considering the half-life value, the flares detected from DO Cep reach maximum energy in longer times, while the geometries of flaring loops or areas get smaller.

\section{Acknowledgments}

The author acknowledges generous allotments of observing time at the Ege University Observatory. I also thank the referee for useful comments that have contributed to the improvement of the paper.

\section{References}

Benz, A. O. \& Güdel, M., 2010, ARA\&A, 48, 241

Contadakis, M. E, Mahmoud, F. M., Mavridis, L. N. \& Stavridis, D., 1982, IBVS, 2088, 1 
Dal, H. A. \& Evren, S., 2010, AJ, 140, 483

Dal, H. A. \& Evren, S., 2011a, AJ, 141, 33

Dal, H. A. \& Evren, S., 2011b, PASJ, 63, 427

Dawson, B. \& Trapp, R. G., 2004, in Basic and Clinical Biostatistics (City: The McGraw-Hill Companies Inc. Press), 61, 134, 245

Doyle, J. G., 1996a, A\&A, 307, 162

Doyle, J. G., 1996b, A\&A, 307, L45

Favata, F., Flaccomio, E., Reale, F., Micela, G., Sciortino, S., Shang, H., Stassun, K. G. \& Feigelson, E. D., 2005, ApJS, 160,469

Gershberg, R. E., 1972, Ap\&SS, 19, 75

Gershberg, R. E., 2005, Solar-Type Activity in Main-Sequence Stars (New York: Springer), 53, 191, 192, 194, 211, 325, 360

Gershberg, R. E. \& Shakhovskaya, N. I., 1983, Ap\&SS, 95, 235

Glebocki, R. \& Gnacinski, P., 2005, yCat., 3244, 0

Green, S. B., Salkind, N. J., \& Akey, T. M., 1999, Using SPSS for Windows: Analyzing and Understanding Data (Upper Saddle River, N.J.: Prentice Hall Press), 50

Grinin, V. P., 1983, Activity in Red-dwarf Stars, Proc. Seventy-first Colloq., Astrophys. Space Sci. Libr.102, Dordrecht: Reidel, 613

Gurzadian, G. A., 1988, ApJ, 332, 183

Hardie, R. H., 1962, in Astronomical Techniques, ed. W. A. Hiltner (Chicago: Univ. Chicago Press), 178a

Haro, G. \& Chavira, E., 1955, BOTT, 21, 3

Haro, G. \& Parsamian, E., 1969, BOTT, 5, 45

Henry, T. J., Walkowicz, L. M., Barto, T. C. \& Golimowski, D. A., 2002, AJ, 123, 2002

Herr, R. B. \& Brcich, J. A., 1969, IBVS, 329, 1

Hudson, H. S. \& Khan, J. I., 1997, ASPC, 111, 135

Imanishi, K., Nakajima, H., Tsujimoto, M., Koyama, K. \& Tsuboi, Y., 2003, PASJ, 55, 653

Ishida, K., Ichimura, K., Shimizu, Y. \& Mahasenaputra, 1991, Ap\&SS, 182, 227

Jenkins, J. S., Ramsey, L. W., Jones, H. R. A., Pavlenko, Y., Gallardo, J., Barnes, J. R. \& Pinfield, D. J., 2009, A\&A, 704, 975

Kirkup, L. \& Frenkel, R. B., 2006, An Introduction to Uncertainty in Measurement (Cambridge: Cambridge University Press)

Kunkel, W., 1967. An optical study of stellar flare. Thesis. Austin, Texas

Lacy, C. H., Moffett, T. J \& Evans, D. S, 1976, ApJS, 30, 85

Lacy, C. H., 1977, ApJS, 34, 479

Landolt, A. U., 1992, AJ, 104, 340

Leto, G., Pagano, I., Buemi, C. S. \& Rodonó, M, 1997, A\&A, 327, 1114
Mavridis, L. N. \& Avgoloupis, S., 1986, A\&A, 154, 171

Meištas, E. G., 2002, High-Speed Three-Channel Photometer (HSTCP) User's Guide, To Molétai version (Vilnius: Astronomical Observatory of Vilnius University)

Moffett, T. J., 1974, ApJS, 29, 1

Motulsky, H., 2007, in GraphPad Prism 5: Statistics Guide, ed. H. Motulsky (San Diego, CA: GraphPad Software Inc. Press), 94, 133

Nicastro, A. J., 1975, IBVS, 1045, 1

Osawa, K., Ichimura, K., Noguchi, T. \& Watanabe, E., 1968, TokAB, No. 180

Oskanian, V. S., 1969, in Non-Periodic Phenomena in Variable Stars, Proc. AUI Coll. No 4., ed. L. Detre (Budapest: Academic Press), 131

Pandey, J. C. \& Singh, K. P., 2008, MNRAS, 387, 1627

Petschek, H. E., 1964, in Proc. of AAS-NASA Symp. on the Physics of Solar Flares, ed. W. N. Hess, NASA SP-50, 425

Pettersen, B. R., 1991, MmSAI, 62, 217

Pettersen, B. R., Coleman, L. A. \& Evans, D. S., 1984, ApJ, 282, 214

Reeves, K. K. \& Warren, H. P., 2002, ApJ, 578, 590

Rodonó, M., 1990, IAUS, 137, 371

Schmitt, J. H. M. M. \& Liefke, C., 2004, A\&A, 417, 651

Skumanich, A. \& McGregor, K., 1986, AdSpR., 6, 151

Söderhjelm, S., 1999, A\&A, 341, 121

Spanier, J. \& Oldham, K. B., 1987, An Atlas of Function (Washington D.C., USA: Hemisphere Publishing Corporation Press), 233

Tamazian, V. S., Docobo, J. A., Melikian, N. D. \& Karapetian, A. A., 2006, PASP, 118,814

Van Den Oord, G. H. J. \& Barstow, M. A., 1988, A\&A, 207, 89

Van Den Oord, G. H. J., Mewe, R. \& Brinkman, A. C., 1988, A\&A, 205, 181

Vandenberg, D. A., Hartwick, F. D. A., Dawson, P. \& Alexander, D. R., 1983, ApJ, 266, 747

Veeder, G. J., 1974, AJ, 79, 702

Vilhu, O., Neff, J. E., \& Walter, F. M., 1986, in New Insight in Astrophysics, ESA SP-263, ed. E. J. Rolfe, 113

Vilhu, O. \& Rucinski, S. M., 1983, A\&A, 127, 5

Walker, A. R., 1981, MNRAS, 195, 1029

Wall, J. W. \& Jenkins, C. R., 2003, in Practical Statistics For Astronomers (Cambridge: Cambridge University Press), 79

Wilson, O.C., 1978, ApJ, 226, 379 